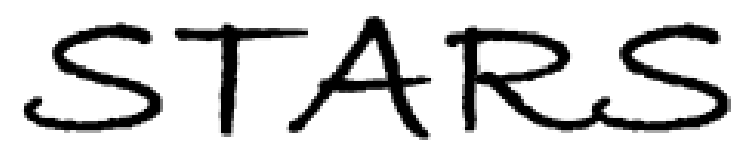

University of Central Florida

STARS

$1-1-2015$

\title{
Thermal modeling and coefficient identification of shape memory polymer nanocomposites structure
}

\author{
He Shen \\ University of Central Florida \\ August Mark \\ University of Central Florida \\ Kenneth Thompson \\ University of Central Florida \\ Yunjun Xu \\ University of Central Florida \\ Fei Liang \\ University of Central Florida \\ Find similar works at: https://stars.library.ucf.edu/facultybib2010 \\ University of Central Florida Libraries http://library.ucf.edu \\ See next page for additional authors
}

This Article is brought to you for free and open access by the Faculty Bibliography at STARS. It has been accepted for inclusion in Faculty Bibliography 2010 s by an authorized administrator of STARS. For more information, please contactSTARS@ucf.edu.

\section{Recommended Citation}

Shen, He; Mark, August; Thompson, Kenneth; Xu, Yunjun; Liang, Fei; Gou, Jihua; and Mabbott, Bob, "Thermal modeling and coefficient identification of shape memory polymer nanocomposites structure" (2015). Faculty Bibliography 2010s. 6798.

https://stars.library.ucf.edu/facultybib2010/6798

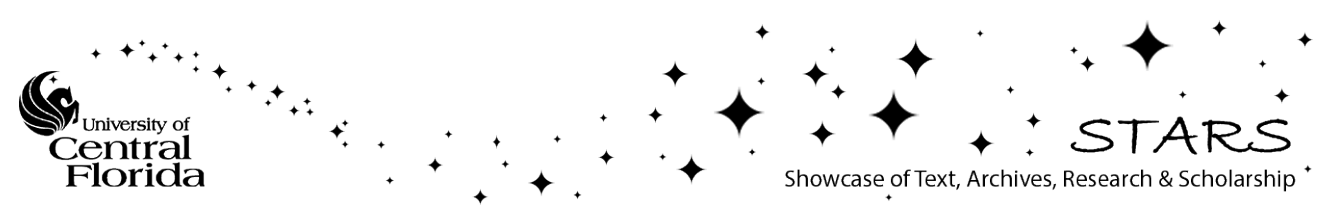


Authors

He Shen, August Mark, Kenneth Thompson, Yunjun Xu, Fei Liang, Jihua Gou, and Bob Mabbott 


\section{Thermal modeling and coefficient identification of shape memory polymer nanocomposites structure}

Cite as: Appl. Phys. Lett. 106, 081907 (2015); https://doi.org/10.1063/1.4913804

Submitted: 08 December 2014 . Accepted: 16 February 2015 . Published Online: 25 February 2015

He Shen, August Mark, Kenneth Thompson, Yunjun Xu, Fei Liang, Jihua Gou, and Bob Mabbott
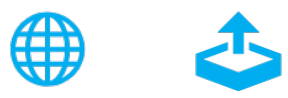

\section{ARTICLES YOU MAY BE INTERESTED IN}

Recovery torque modeling of carbon fiber reinforced shape memory polymer nanocomposites Applied Physics Letters 103, 201903 (2013); https://doi.org/10.1063/1.4829937

Artificial muscles made of chiral two-way shape memory polymer fibers

Applied Physics Letters 109, 183701 (2016); https://doi.org/10.1063/1.4966231

An investigation of a thermally steerable electroactive polymer/shape memory polymer hybrid actuator

Applied Physics Letters 108, 062901 (2016); https://doi.org/10.1063/1.4941802

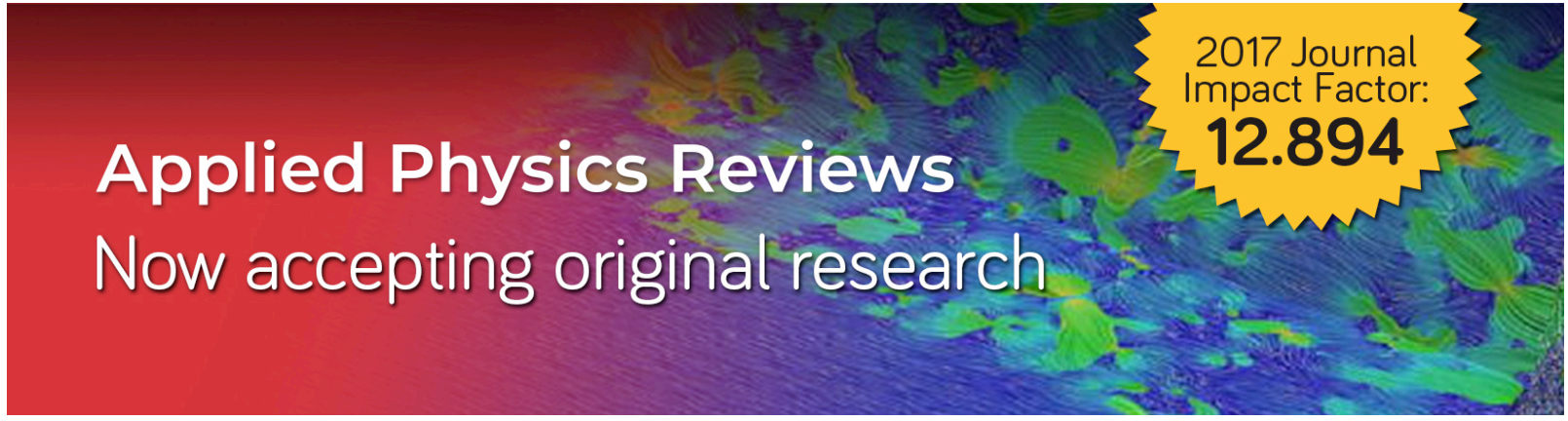




\title{
Thermal modeling and coefficient identification of shape memory polymer nanocomposites structure
}

\author{
He Shen, ${ }^{1}$ August Mark, ${ }^{1}$ Kenneth Thompson, ${ }^{1}$ Yunjun Xu, ${ }^{1, a)}$ Fei Liang, ${ }^{2}$ Jihua Gou, ${ }^{2}$ \\ and Bob Mabbott ${ }^{3}$ \\ ${ }^{1}$ Dynamics and Controls Laboratory, Department of Mechanical and Aerospace Engineering, \\ University of Central Florida, Orlando, Florida 32816, USA \\ ${ }^{2}$ Composite Materials and Structures Laboratory, Department of Mechanical and Aerospace Engineering, \\ University of Central Florida, Orlando, Florida 32816, USA \\ ${ }^{3}$ HB Polymer Company, Sarasota, Florida 34243, USA
}

(Received 8 December 2014; accepted 16 February 2015; published online 25 February 2015)

\begin{abstract}
An increasing amount of research has been conducted on developing new shape memory polymer nanocomposite structures. Estimating thermal properties of these types of structures is a crucial step towards their successful application. In this paper, a finite element based thermal model is proposed, of which the coefficients are identified using a nonlinear programming method in a nested loop. The model and identification results are validated in three material samples. (C) 2015
\end{abstract} AIP Publishing LLC. [http://dx.doi.org/10.1063/1.4913804]

Shape memory polymers (SMPs) are polymeric smart materials that can recover to their permanent shapes under various stimuli. ${ }^{1-5}$ Among these stimulation methods, electric actuation using resistive heat has recently received much attention. ${ }^{1,6}$ However, this method can introduce nonuniform thermal loading in manufactured SMP nanocomposite structures (SMPNSs) resulting in over-heating in certain areas. ${ }^{7}$ Meanwhile, the thermal properties of SMPNSs for new applications are often not provided or cannot be readily measured. To date, many different approaches have been used to quantify thermal properties. Laser reflectance thermometry is used to measure the thermal conductivity in thin films, ${ }^{8}$ thermal diffusivity in polymer matrix composites can be obtained through a laser flash apparatus, ${ }^{9}$ while differential scanning calorimetry can measure the glass transition temperature in SMPs. ${ }^{10}$ Micro-electrode structures can also be used to measure the thermal conductivity in thin films with precisely designed assistive micro-electron structures. ${ }^{11}$ However, the above mentioned methods require expensive equipment and/or multiple testing devices to measure multiple thermal properties. Thus, a low cost approach to predict simultaneously multiple thermal properties is necessary for rapidly designing and customizing SMPNSs for applications.

This paper presents an indirect method of estimating thermal properties for electrically actuated SMPNSs. An approximate thermal model is derived based on a finite element approach, in which the element size is determined by the pixel size of a thermal imager. Both conduction and convection are considered. Nonlinear programming in a nested loop is used to identify the thermal coefficients and glass transition temperature.

Figure 1 shows the SMPNS schematic. It is composed of an SMP matrix with a thin layer of carbon nanopaper (CNP) and a layer of distributed carbon fibers (CFs) meant to improve electrical conductivity and mechanical strength, respectively. The carbon nanofibers (CNFs) in the CNP,

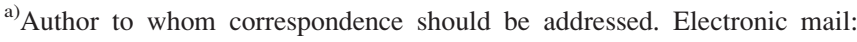
yunjun.xu@ucf.edu
}

from Applied Sciences, measure $100 \mathrm{~nm}$ in diameter and $20-200 \mu \mathrm{m}$ in length, and have a non-bulk density of $1.9 \mathrm{~g} /$ $\mathrm{ml}$ at $25^{\circ} \mathrm{C}$.

Table I lists the thermal conductivity, specific heat, volume and mass percentages of SMP resin, CNF paper, and carbon fiber for three samples. The sizes of the samples (length, width, and thickness) are $(77.8,32.0$, and 2.30$) \mathrm{mm}$ (78.4, 31.8, and 2.30) $\mathrm{mm}$, and $(80.6,36.4$, and 2.22$) \mathrm{mm}$, respectively.

A finite element model is used to describe the heat transfer between the point of interest and its surroundings. In Fig. 2 , we limit our region of interest to a single element $P_{0}$ surrounded by four adjacent elements $P_{i}, i=1,2, \ldots, 4$. The element size can range between one or several pixels in thermal images. Conduction occurs between the element of interest and the four adjacent elements. Convection occurs between the upper and lower surfaces of the SMPNS and the ambient atmosphere. Energy is supplied to the SMPNS through electrical resistive heat. Radiation from the SMPNS to the surroundings is relatively small and omitted here.

Conduction between element $P_{0}$ and the adjacent elements is given by Fourier's Law ${ }^{16}$ as

$$
\dot{Q}_{1}=C_{1}\left(A_{C} / \Delta L\right) \sum_{i=1}^{4}\left[T(t)-T_{i}(t)\right]
$$

where $C_{1}, A_{C}, \Delta L, T$, and $T_{i}, i=1, \ldots, 4$ are the thermal conductivity coefficient, cross section area, length of element $P_{0}$, temperature of element $P_{0}$, and temperatures of adjacent

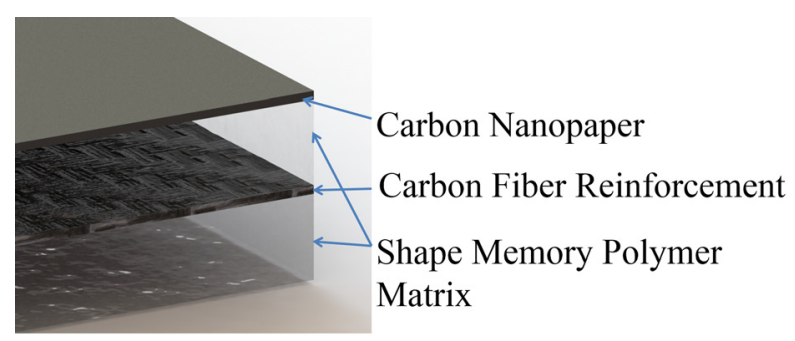

FIG. 1. The structure of SMPNS. 
TABLE I. Properties of composites in samples. ${ }^{2,12-15}$

\begin{tabular}{lccc}
\hline \hline & SMP resin & CNF paper & CF \\
\hline Conductivity $(\mathrm{W} / \mathrm{m} \cdot \mathrm{K})$ & 0.12 & 2000 & 15 \\
Specific heat $(\mathrm{J} / \mathrm{g} \cdot \mathrm{K})$ & $2.5-3.3$ & 0.93 & 0.8 \\
Sample 1 vol. \% & $69.37 \%$ & $2.80 \%$ & $27.83 \%$ \\
(mass \%) & $(48.39)$ & $(5.22)$ & $(46.39)$ \\
Sample 2 vol. \% & $70.31 \%$ & $1.87 \%$ & $27.83 \%$ \\
(mass \%) & $(50.65)$ & $(3.45)$ & $(45.90)$ \\
Sample 3 vol. \% & $67.62 \%$ & $3.54 \%$ & $28.82 \%$ \\
$($ mass \%) & $(37.92)$ & $(7.51)$ & $(54.58)$ \\
\hline \hline
\end{tabular}

elements, respectively. From Refs. 2 and 12, the thermal conductivity coefficient varies by less than $10 \%$ with respect to temperature. Thus, we assume $C_{1}$ to be constant in the approximate model.

The convection to surrounding atmosphere follows the model discussed in Ref. 16 as:

$$
\begin{aligned}
\dot{Q}_{2} & =\dot{Q}_{2, \text { top }}+\dot{Q}_{2, \text { bot }} \\
& =\frac{C_{2} A_{s}}{L^{1 / 4}}\left[0.54\left(T-T_{\infty}\right)^{5 / 4}+0.27\left(T+\Delta T-T_{\infty}\right)^{5 / 4}\right],
\end{aligned}
$$

where $A_{S}, L, T_{\infty}, \Delta T$, and $C_{2}$ are the surface area of element $P_{0}$, sample characteristic length, ambient temperature, temperature difference between the top and bottom surfaces, and remaining unknown terms used to calculate the coefficients of convection $h$ for both surfaces. The dimensionless numbers 0.54 and 0.27 are the constants associated with free convection for hot surfaces facing upward and downward. ${ }^{16}$ While $C_{2}$ depends on the ambient and thermal boundary layer temperatures and $\Delta T$ varies over time, the change in both parameters is not significant, and they are assumed constant for the approximate model.

The temperature change of $P_{0}$ is modeled as ${ }^{16}$

$$
\dot{Q}_{3}=C_{3} m_{E} \dot{T}
$$

where $C_{3}$ is the specific heat and $m_{E}$ is the mass of element $P_{0}$. As shown in Refs. 2 and 12, the heat capacity of polymeric materials increases exponentially within $\pm 15^{\circ} \mathrm{C}$ of the glass transition temperature, and is mostly constant outside this region. This behavior can be approximated by

$$
C_{3}= \begin{cases}C_{3,1} \exp \left[C_{3,2}\left(T-T_{G}\right)\right]+C_{3,4} & \text { if } T \leq T_{G} \\ C_{3,1}\left\{2-\exp \left[C_{3,3}\left(T_{G}-T\right)\right]\right\}+C_{3,4} & \text { if } T>T_{G} .\end{cases}
$$

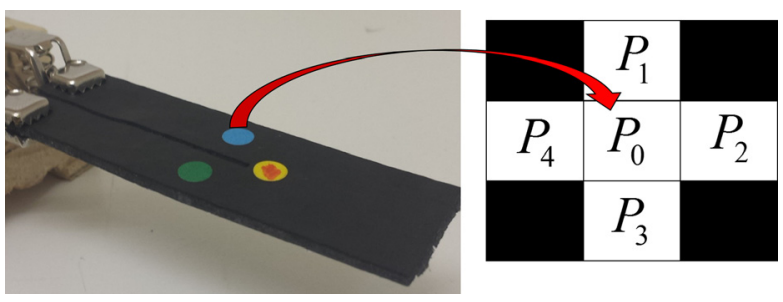

FIG. 2. One test sample with element formulation.
The constants $C_{3, i}, i=1,2,3,4$ are to be identified. Equation (4) can generate specific heat profiles that approximately match the trend of those shown in Refs. 2 and 12 for temperatures up to $100{ }^{\circ} \mathrm{C}$. Assuming the heat generation over the SMPNS surface is uniform, the energy input to element $P_{0}$ is given by Ohm's $\mathrm{Law}^{17}$ as

$$
\dot{Q}_{G}=\left(A_{S} / A\right) U I
$$

where $A_{S}, A, U$, and $I$ are the surface area of element $P_{0}$, top surface area of SMPNS, voltage, and current, respectively. Thermographic observations taken during experiments show that the heat generation is non-uniform and dependent on sample geometry. The temperature mismatch due to the assumption of uniform power generation can be compensated by the coefficients identified.

According to the law of conservation of energy

$$
\dot{Q}_{G}=\dot{Q}_{1}+\dot{Q}_{2}+\dot{Q}_{3},
$$

in which the unknown coefficients $\boldsymbol{x}$, including $C_{1}, C_{2}$, $C_{3, i}, i=1,2,3,4, \Delta T$, and $T_{G}$, are identified through two nonlinear constrained optimizations in a nested loop.

The inner loop optimization searches for a solution that can minimize the error between the predicted and measured temperature profiles for all the experiments. Nonlinear programming solvers can compute many local minima; therefore, an outer loop optimization is applied to control the bounds limiting the unknown coefficients. In this approach, the identified coefficients can be consistent among different experiments.

The inner loop finds the optimal $\boldsymbol{x}^{j}$ to match experiment $j, j=1, \ldots, N$, where $N$ is the number of experiments being analyzed. The inner loop performance index for experiment $j, J_{i n}^{j}$, is

$$
J_{i n}^{j}=\left(\sum_{k=1}^{N^{j}}\left|T_{k}^{j}-\hat{T}_{k}^{j}\right|\right) / N^{j}
$$

where $N^{j}, T_{k}^{j}$, and $\hat{T}_{k}^{j}$ are the number of time nodes, measured temperature at time node $k$, and the propagated temperature with identified $x^{j}$.

$\boldsymbol{x}^{j}$ in the inner loop optimization is constrained by $\boldsymbol{x}_{L B} \leq \boldsymbol{x}^{j} \leq \boldsymbol{x}_{U B}$, and those bounds are optimized in the outer loop with the following performance index:

$$
J_{\text {out }}=\sum_{l=1}^{8}\left(\sigma_{l} / x_{\max , l}\right)+\sum_{j=1}^{N} P_{j}
$$

where $\sigma_{l}$ and $x_{\max , l}$ are the standard deviation and maximum value of identified coefficient $\boldsymbol{x}_{l}$ across all $N$ experiments. $P_{j}$ is the penalty: $P_{j}=1000 J_{i n}^{j}$ if $J_{i n}^{j}$ is above a user-defined threshold; otherwise, $P_{j}=0$.

The bounds on $\boldsymbol{x}_{L B}$ and $\boldsymbol{x}_{U B}$ must satisfy $D \leq \boldsymbol{x}_{U B}$ $-\boldsymbol{x}_{L B} \leq E, \boldsymbol{x}_{U B} \leq \overline{\boldsymbol{x}}_{U B}$, and $\boldsymbol{x}_{L B} \geq \underline{\boldsymbol{x}}_{L B}$, in which $D$ and $E$ are the minimum and maximum separations between $x_{L B}$ and $\boldsymbol{x}_{U B}$, while $\overline{\boldsymbol{x}}_{U B}$ and $\underline{\boldsymbol{x}}_{L B}$ are the upper and lower bounds placed on $\boldsymbol{x}_{L B}$ and $\boldsymbol{x}_{U B}$. These bounds are found from the known characteristics of SMPNS constituents, as shown in Table I. $\underline{\boldsymbol{x}}_{L B}$ and $\overline{\boldsymbol{x}}_{U B}$ for $C_{1}$ are chosen based on the Lewis and Nielson model. ${ }^{18} \underline{\boldsymbol{x}}_{L B}$ and $\overline{\boldsymbol{x}}_{U B}$ for $C_{2}$ depend on the remaining coefficients from the free convection model 


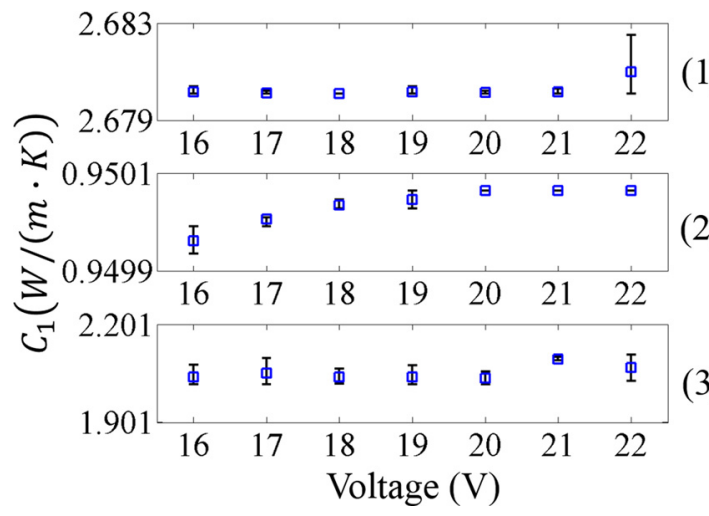

FIG. 3. Conduction coefficient $C_{1}$ : (1) sample 1, (2) sample 2, and (3) sample 3.

discussed in Ref. 16, which depend on the ambient temperature and the thermal boundary layer temperature. $\underline{x}_{L B}$ and $\overline{\boldsymbol{x}}_{U B}$ for $C_{3,1}$ and $C_{3,4}$ are determined by assuming that the specific heat is proportional to the volume percentage of each composite material, and then adding a margin to each estimated value. $\underline{\boldsymbol{x}}_{L B}$ and $\overline{\boldsymbol{x}}_{U B}$ for $C_{3,2}$ and $C_{3,3}$ are chosen to match the specific heat profile, as shown in Refs. 2 and 12. $\underline{\boldsymbol{x}}_{L B}$ and $\overline{\boldsymbol{x}}_{U B}$ for $\Delta T$ are arbitrarily chosen to be $-10^{\circ} \mathrm{C}$ and $0^{\circ} \mathrm{C} . T_{G}$ is known to be approximately $60^{\circ} \mathrm{C}$, so margins of $\pm 1^{\circ} \mathrm{C}$ are used.

The temperature and current are measured in real-time using a thermal imager and a current sensor. The MATLAB function fmincon is used as the optimization solver. It finds proper thermal coefficients to match the identified temperature profiles with the measured ones. Three samples were tested under voltage inputs from $16 \mathrm{~V}$ to $22 \mathrm{~V}$ with increments of $1 \mathrm{~V}$. Under each voltage, five tests were carried out. Some obvious outliers are omitted.

In Figs. 3-5, the squares show the average of identified coefficients for $C_{1}, C_{2}$, and $\Delta T$, and the error bars show the variation in these coefficients, which are shown to be small. Fig. 3 shows that the $C_{1}$ values are roughly $2.68 \mathrm{~W} /(\mathrm{m} \cdot K)$, $0.95 \mathrm{~W} /(\mathrm{m} \cdot \mathrm{K})$, and $2.0 \mathrm{~W} /(\mathrm{m} \cdot \mathrm{K})$ for samples 1,2 , and 3 , respectively. While we expect sample 3 to have a higher conduction compared to sample 1, given the increased CNF mass percentage, this discrepancy can be explained by the nonuniformity experienced in the carbon nanofibers. A sample with more nanofibers can be less conductive than a sample

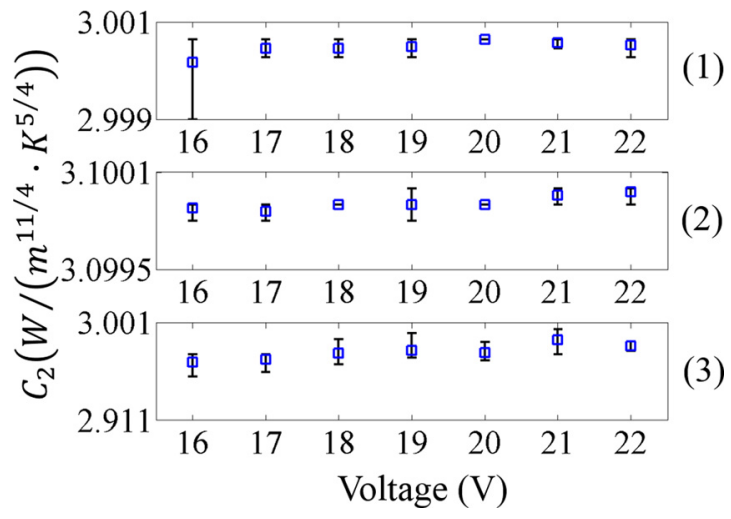

FIG. 4. Convection coefficient $C_{2}$ : (1) sample 1, (2) sample 2, and (3) sample 3.

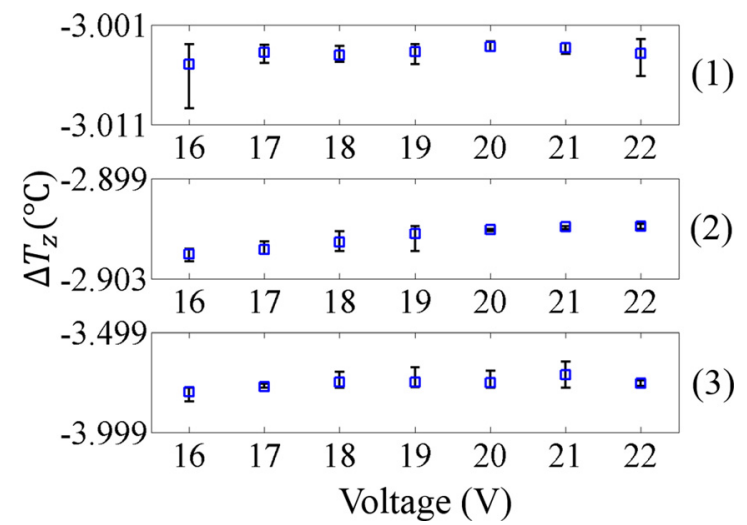

FIG. 5. Temperature difference between both surfaces $\Delta T$ : (1) sample 1, (2) sample 2, and (3) sample 3.

with longer and more aligned nanofibers. Fig. 4 shows that the $C_{2}$ values are approximately $3.0 \mathrm{~W} /\left(\mathrm{m}^{11 / 4} \cdot K^{5 / 4}\right), 3.1$ $W /\left(m^{11 / 4} \cdot K^{5 / 4}\right)$, and $3.0 \mathrm{~W} /\left(m^{11 / 4} \cdot K^{5 / 4}\right)$ for samples 1,2 , and 3 , respectively. All experiments were performed under the same ambient conditions, so this similarity is expected. Fig. 5 shows that the $\Delta T$ values for three samples are approximately $-3.0^{\circ} \mathrm{C},-2.9^{\circ} \mathrm{C}$, and $-3.7^{\circ} \mathrm{C}$ for samples 1,2 , and 3 , respectively. This is expected, as the temperature difference is due to conduction through the SMP resin, and these samples have similar SMP contents. Fig. 6 shows the identified $C_{3}$ for the samples over temperature. It can be seen that the specific heats of samples 1 and 2 are very close, which are expected from the similar SMP resin and CF volume percentages. It can also be seen that the specific heats undergo the most change in the region of $\pm 15^{\circ} \mathrm{C}$ of the $T_{G}$, which agrees with previously reported results. ${ }^{2,12}$ The decrease in specific heat of sample 3 can also be explained by the lower SMP mass percentage in sample 3 compared to samples 1 and 2 . The measured temperature profiles and the predicted profiles using the identified thermal properties match well for all the experiments. In Fig. 7 (19V experiments for all three samples), example identification results show that the propagated temperature profiles match with the experimental data.

Instead of measuring the thermal properties separately with different and expensive instruments, this paper presents an indirect method of estimating thermal properties for electrically actuated SMPNSs using the data measured via low cost, off-the-shelf devices (e.g., current sensor and thermal

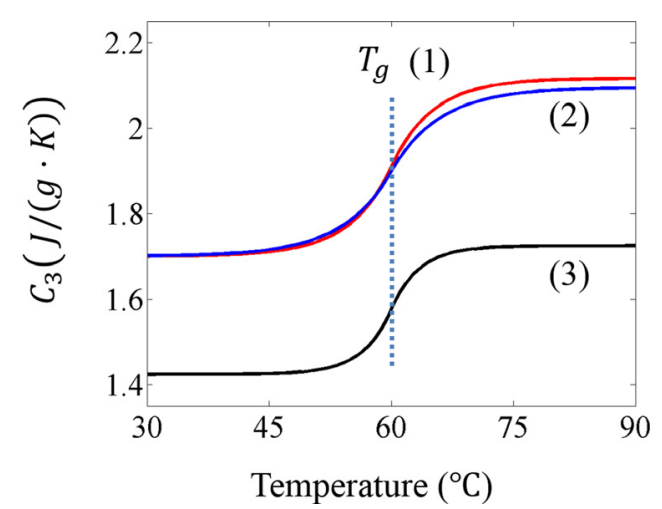

FIG. 6. Specific heat $C_{3}$ vs. temperature: (1) sample 1, (2) sample 2, and (3) sample 3 . 


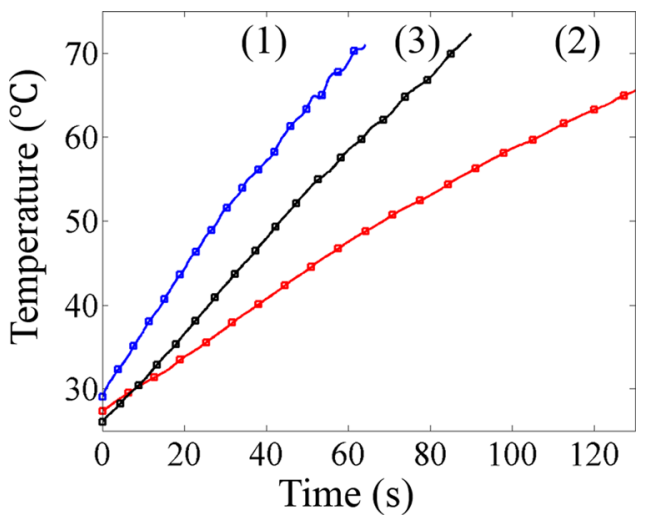

FIG. 7. Predicted ("line") and measured ("dotted") temperature profiles in a $19 \mathrm{~V}$ case: (1) sample 1, (2) sample 2, and (3) sample 3.

imager). In the thermal model, convection and conduction are considered. Based on this model, a two-layer optimization is formulated and solved for the parameters. Three samples are tested. The temperature profiles predicted using those identified thermal coefficients match well with the measured ones. It is expected that the proposed method can significantly accelerate the design and application of SMPNSs.

Thanks are given to the HB Polymer, Inc., and the Florida High-Tech Corridor Program for financial support.
The authors would also like to thank John Sparkman for making several samples.

${ }^{1}$ J. Gou, F. Liang, Y. Xu, and B. Mabbott, Appl. Mech. Mater. 423, 97 (2013).

${ }^{2}$ B. Weidenfeller and M. Anhalt, J. Thermoplast. Compos. Mater. 27, 895 (2014).

${ }^{3}$ A. Lendlein, H. Jiang, O. Junger, and R. Langer, Nature 434, 879 (2005).

${ }^{4}$ K. Yu, Y. Liu, and J. Leng, RSC Adv. 4, 2961 (2014).

${ }^{5}$ C. Zhang, H. Wei, Y. Liu, H. Tan, and Z. Guo, High Perform. Polym. 24, 702 (2012).

${ }^{6}$ H. Shen, Y. Xu, F. Liang, J. Gou, and B. Mabbott, Appl. Phys. Lett. 103, 201903 (2013).

${ }^{7}$ H. Lu, F. Lang, J. Gou, J. Leng, and S. Du, Smart Mater. Struct. 23, 085034 (2014).

${ }^{8}$ K. Goodson, O. Käding, M. Rösler, and R. Zachai, J. Appl. Phys. 77, 1385 (1995).

${ }^{9}$ B. Weidenfeller, M. Anhalt, and S. Kirchberg, J. Appl. Phys. 112, 093513 (2012).

${ }^{10} \mathrm{~J}$. Champagne, Ph.D. dissertation, Louisiana State University, 2013.

${ }^{11}$ D. Chu, M. Touzelbaev, K. Goodson, S. Babin, and R. Pease, J. Vac. Sci. Technol., B 19(6), 2874 (2001).

${ }^{12}$ M. Razzaq and L. Frormann, Polym. Compos. 28, 287 (2007).

${ }^{13}$ J. Ullbrand, J. Cordoba, J. Tamayo-Ariztondo, M. Elizalde, M. Nygren, J. Molina-Aldareguia, and M. Oden, Compos. Sci. Technol. 70, 2263 (2010).

${ }^{14}$ H. Lu, Y. Liu, J. Gou, J. Leng, and S. Du, Int. J. Smart Nano Mater. 1, 2 (2010).

${ }^{15}$ V. Cecen, I. Tavman, M. Kok, and Y. Aydogdu, Polym. Compos. 30, 1299 (2009).

${ }^{16}$ A. Bejan, Heat Transfer (Wiley, New York, 1993).

${ }^{17}$ J. Irwin, A Brief Introduction to Circuit Analysis (Wiley, New York, 2003).

${ }^{18}$ R. Pal, Composites Part A 39, 718 (2008). 